\title{
ARTICLE
}

\section{A Probabilistic Risk Assessment for Field Radiography Based on Expert Judgment and Opinion}

\author{
Han-Ki JANG ${ }^{1}$, Hyung-Joon RYU ${ }^{1}$, Ji-Young KIM ${ }^{1}$, Jai-Ki LEE ${ }^{1 *}$ and Kun-Woo CHO ${ }^{2}$ \\ ${ }^{1}$ Nuclear Engineering, Hanyang Univ., 17 Haengdang-dong, Seongdong-gu, Seoul,133-791, Korea \\ ${ }^{2}$ Korea Institute of Nuclear Safety, 34 Gwahak-ro, Yuseong-ku, Daejon, 305-338, Korea
}

\begin{abstract}
A probabilistic approach was applied to assess radiation risk associated with the field radiography using gamma sources. The Delphi method based on the expert judgments and opinions was used in the process of characterization of parameters affecting risk, which are inevitably subject to large uncertainties. A mathematical approach applying the Bayesian inferences was employed for data processing to improve the Delphi results. This process consists of three phases: (i) setting prior distributions, (ii) constructing the likelihood functions and (iii) deriving the posterior distributions based on the likelihood functions. The approach for characterizing input parameters using the Bayesian inference is provided for improved risk estimates without intentional rejection of part of the data, which demonstrated utility of Bayesian updating of distributions of uncertain input parameters in PRA (Probabilistic Risk Assessment). The data analysis portion for PRA in field radiography is addressed for estimates of the parameters used to determine the frequencies and consequences of the various events modeled. In this study, radiological risks for the worker and the public member in the vicinity of the work place are estimated for field radiography system in Korea based on two-dimensional Monte Carlo Analysis (2D MCA).
\end{abstract}

\section{KEYWORDS: Probabilistic risk assessment, expert judgment and opinion, Delphi method, Bayesian inference,} tow-dimensional Monte Carlo analysis

\section{Introduction}

Radiation risk can be calculated using by crisp estimates of the exposure variables (e.g., source term, exposure time, distance, exposure frequency). The parameters of the various risk models used in the probabilistic Risk Assessment (PRA) are not exactly known because of the scarcity of data. Therefore, the expert role provides valuable information through his or her decision within the framework of the availability of the data which are uncertain and limited, but certainly needed for the analysis. Applicable areas are diverse, including nuclear engineering, aerospace, various types of forecasting (economic, technological, meteorology cal, and snow avalanches), military intelligence, seismic risk, and environmental risk from toxic chemicals. ${ }^{1,2)}$

PRA is a tool for quantitative estimation of risk and associated uncertainties. The prevailing method in PRA is Monte Carlo analysis (MCA), which is a means of quantifying uncertainty of variability in a probabilistic framework using computer simulation. MCA methodology, as well as its application to risk analysis, has been reviewed and detailed in Cullen and Frey(1999), Morgan and Henrion (1990), and elsewhere., ${ }^{3,4)}$ Furthermore two-dimensional Monte Carlo analysis (2D MCA) is a natural extension of one-dimensional Monte Carlo analysis (1D MCA $)^{5-8)}$, which can provide information on uncertainty and variability in the estimates.

In this study, radiological risks for the worker and member of the public in the vicinity of the workplaces of

*Corresponding Author, Tel No:+82-2-2220-0571, Fax No:

+82-2-2296-3690, E-mail: jakilee@hanyang.ac.kr

(C) Atomic Energy Society of Japan field radiography in Korea are estimated based on the 2D MCA. The data analysis portion for PRA is addressed for estimates of the parameters used to determine the frequencies and consequences of the various tasks and events involved in the job. The Delphi survey and the Bayesian update technique are employed in characterizing uncertain variables.

\section{Materials and Method}

In determination of variables and their values characterizing risk associated with field radiography tasks, the Delphi method based on expert judgment was employed. Bayesian approach was used to solve the problem of behavioral approaches for Delphi technique. The batch fit function of Crystalball, which provides optimized distributions of variables using the Chi-square testing, is used to set distribution of input variables. ${ }^{9}$ Uncertainties in the risk results were analyzed by applying 2D MCA based

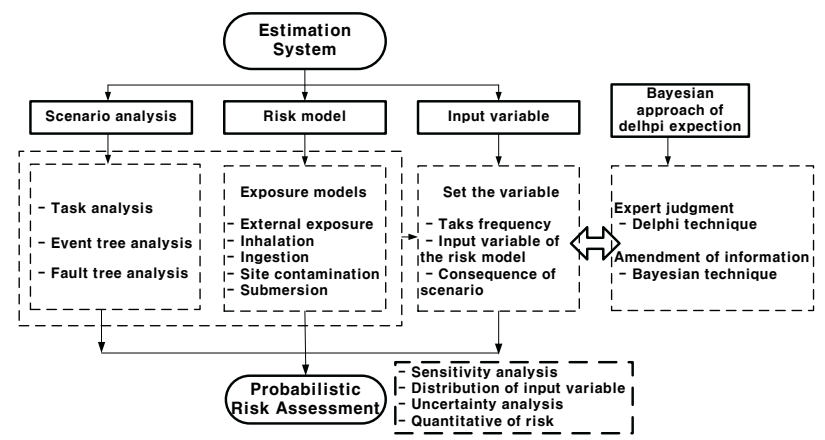

Fig. 1 The brief flow chart to estimate radiological risk assessment of field radiography. 
on the probabilistic inference. The brief flow chart to estimate radiological risk assessment of using task in the field radiography is shown in Fig. 1.

\section{Delphi Method}

The Delphi method is by far the most known method for eliciting and synthesizing expert opinions ${ }^{10,11)}$ and has been proven as a popular tool in information systems research for identifying and prioritizing issues for managerial decision-making. ${ }^{12)}$ A three-stage Delphi survey has been tried out for this work. Twenty expert panel members for this survey divided into two groups of equal sizes; one from a regulatory institution, the Korea Institute of Nuclear Safety (KINS), and one from companies doing field radiography works. The Delphi questionnaire consisted of the general questions for ensuring the professionalism of the experts and the detailed questions for determining the factors needed for risk assessment.

\section{Bayesian Update}

The expert judgment information formulates a prior distribution that is combined with the sample data and its distribution by applying Bayes theorem to form a posterior distribution that reflects a composite of all the available information. The major disadvantage of this approach is that probability distribution functions are needed for both the expert judgment data and for the existing data. The process of combining these distributions can be mathematically difficult. Instead, the Markov Chain Monte Carlo (MCMC) simulation approach was used in calculating the posterior result with aid of the Crystalball's batch fit function, which provides optimized distributions of variables using the chi-squared Goodness-of Fit statistics. The WinBUGS package based on the MCMC method was used for deriving the posterior distribution. ${ }^{13)}$

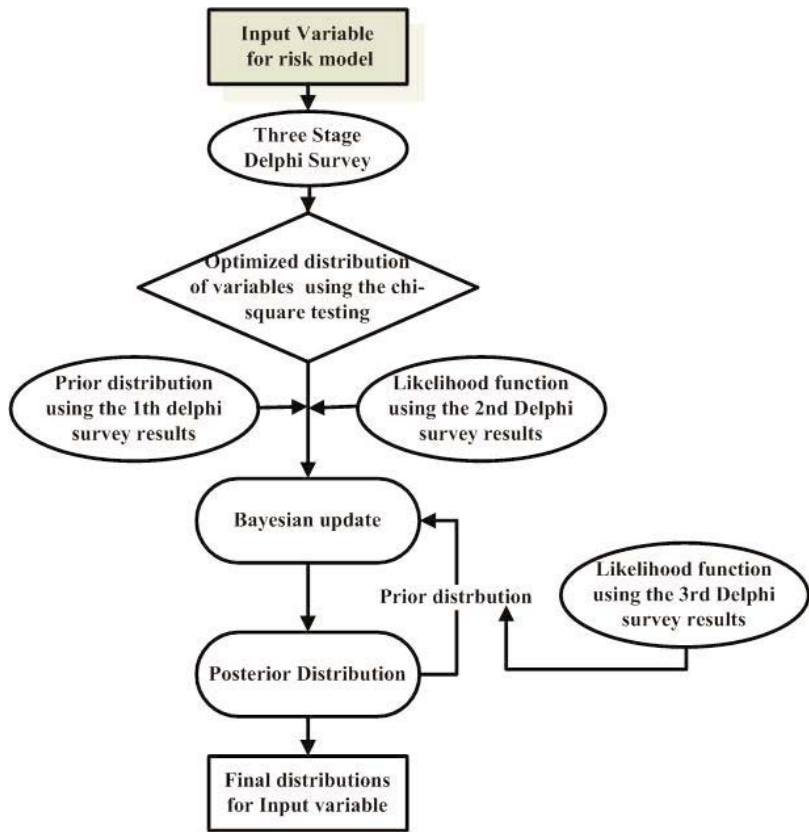

Fig. 2 Bayesian update flow chart.
Updating based on the Bayesian inferences was employed for data processing to improve the Delphi results. This process consists of three phases: (i) quantifying prior distribution, (ii) constructing likelihood function and (iii) deriving posterior distribution based on likelihood function incorporated with the prior distribution. The flow chart of Bayesian update is shown in Fig. 2.

\section{Two-dimensional Monte Carlo analysis}

The 2D MCA is often used in risk analysis accompanying an uncertainty analysis that requires variability to be distinguished from other types of uncertainty ${ }^{14-16)}$. The probability density functions (PDFs) used to describe the variability in the model has some certain degree of uncertainties. The 2D MCA addresses two types of variable; U-type in relation to uncertainty and V-type in relation to variability. The number of runs used was equal to 10,000 for the inner loop calculations for variability and 250 for outer loop calculations for uncertainty, which generated a total of 250 cumulative distribution functions (CDFs).

\section{Event Tree}

The event trees for normal using task and for accidents were constructed by taking into consideration of expected incidence factors of exposure events obtained through consultation to and interviews with workers and experts in this field. The event tree for normal using only is illustrated in Fig. 3.

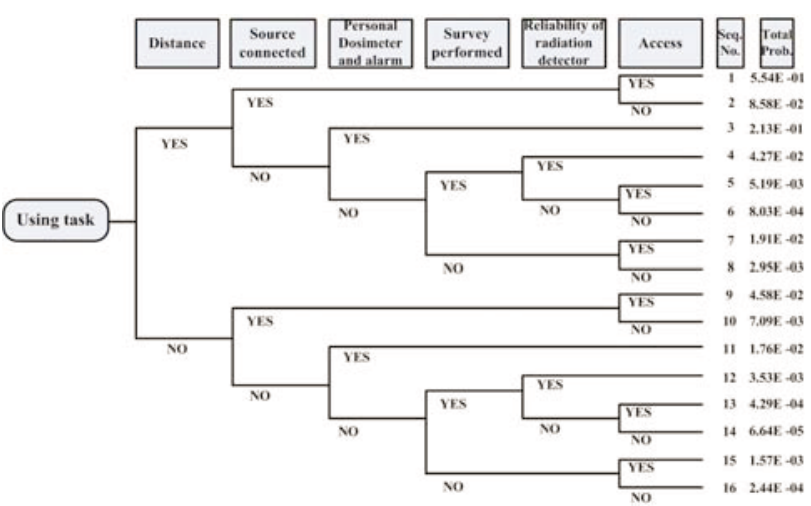

Fig. 3 Event tree and probability for Using task in field radiography.

\section{Results and Discussion}

The input data of 110 were obtained through the three-stage Delphi survey. The results of risk estimates based on 1D MCA using the Delphi panel responses for tasks are shown in Fig. 4. Generally, conservative risk estimates are obtained with the input from experts in the regulatory institution (KINS) compared with those with input from experts from companies.

The overall risks after the Bayesian updating of the inputs are compared with both those without updating $\left(3^{\text {rd }}\right.$ Delphi survey) and those resulted from the estimation employing 

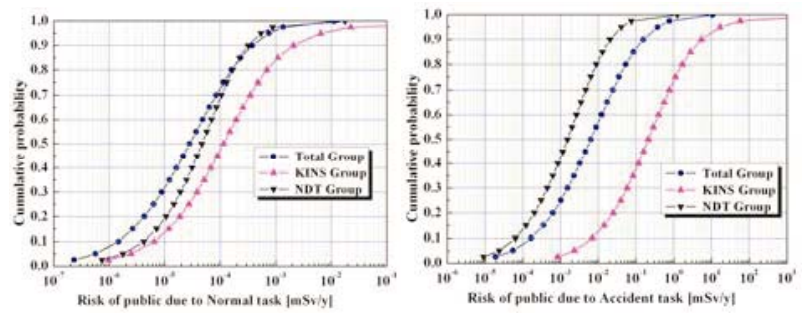

(a) Public
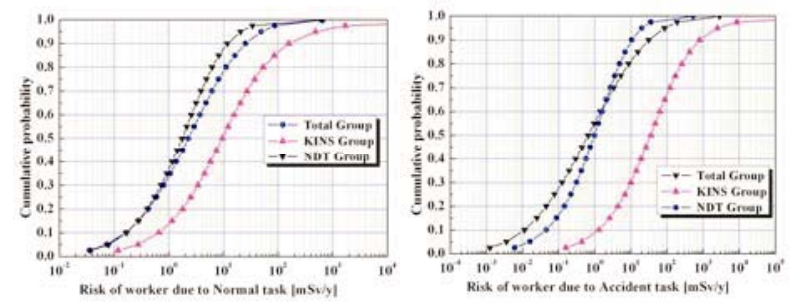

(b) Worker

Fig. 4 Risk from assessment using Delphi panel response in field radiography: (a) public (b) worker due to normal task and accident.

data within the $95 \%$ confidence interval at the third-stage Delphi survey.

It is noted in Fig.4 that the CDFs without updating show unrealistically low and high values of dose in the lower and upper tails.

On the other hand, the risks applying the Bayesian updating in Fig.5 well agree with the risk reflecting 95\% confidence interval. The latter, however, suffers arbitrary rejection of some collected data.

Varieties of meaningful information can be extracted from the results of 2D MCA. From the $250 \mathrm{CDFs}$ generated through 2D MCA, plots of the $90 \%$ confidence intervals for the median were made to show the probabilities of and to provide the comparison between 2D MCA and 1D MCA
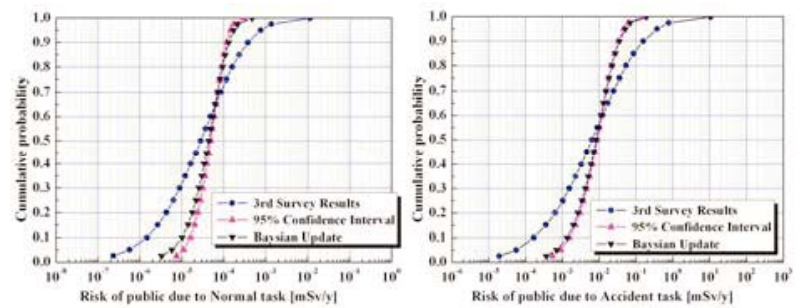

(a) public
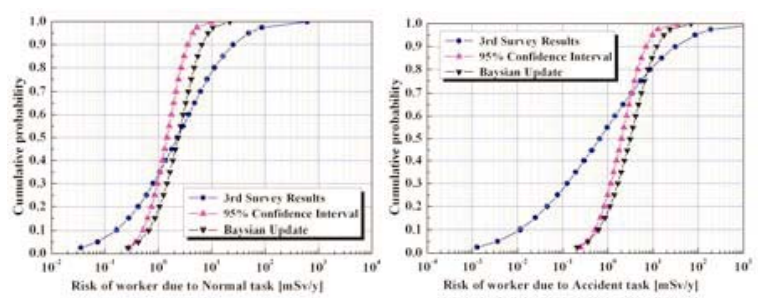

(b) worker

Fig. 5 Risk distributions from different characterizing methods of input variable: (a) public (b) worker due to normal task and accident. results, and this is given in Fig. 6.

Confidence limits for the $2.5^{\text {th }}, 5^{\text {th }}, 10^{\text {th }}, 25^{\text {th }}, 50^{\text {th }}, 75^{\text {th }}$, $90^{\text {th }}$, and the $97.5^{\text {th }}$ percentiles of the radiological risk for the
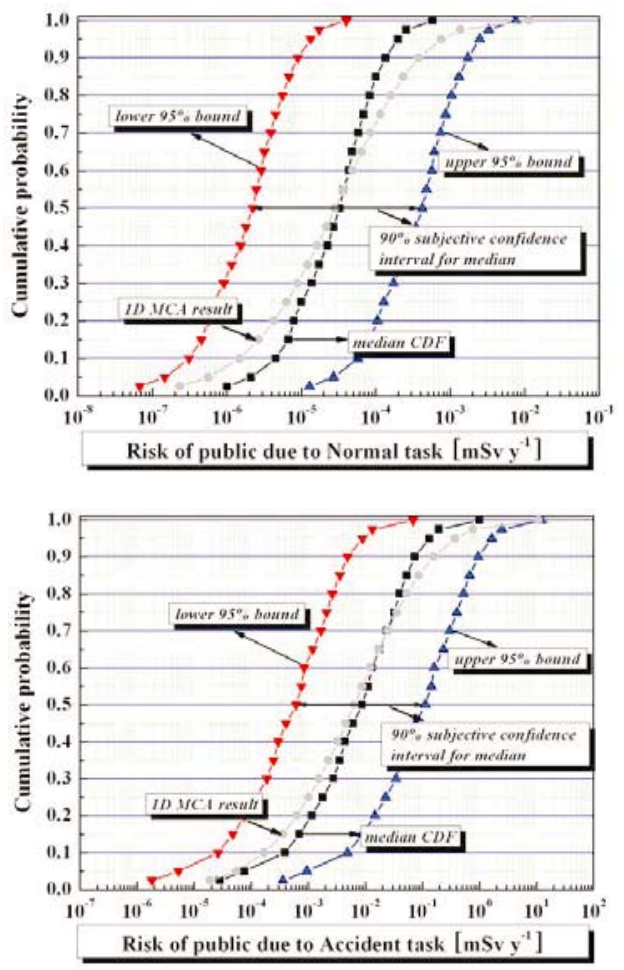

(a) Public
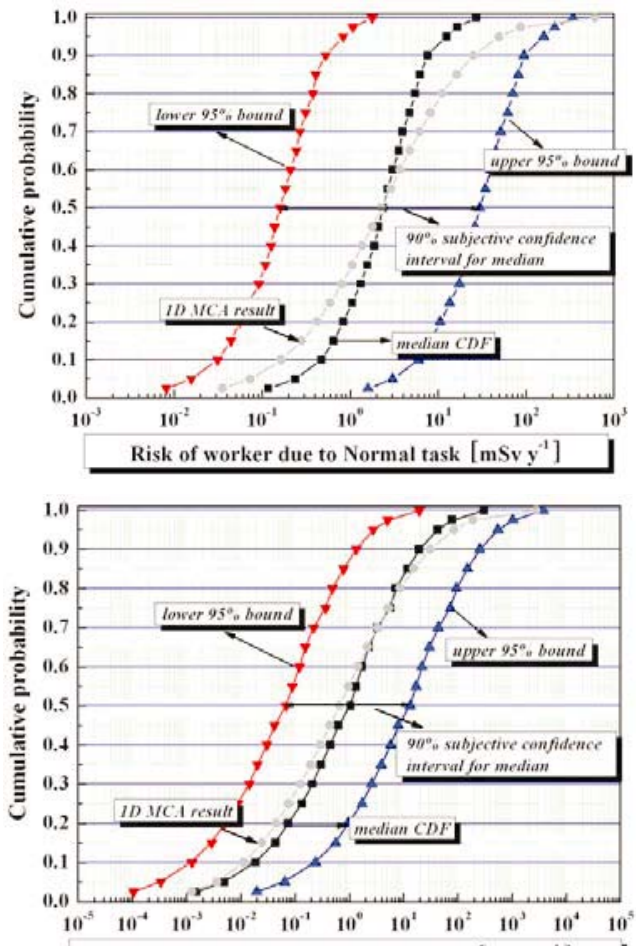

Risk of worker due to Accident task [mSv y ${ }^{4}$ ]

(b) Worker

Fig. 6 90\% confidence interval of risk: (a) public (b) worker due to normal task and accident. 


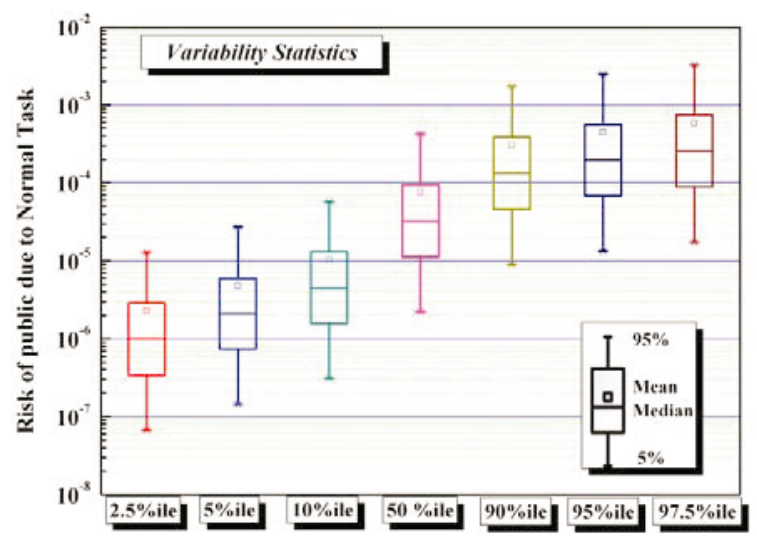

(a) Public

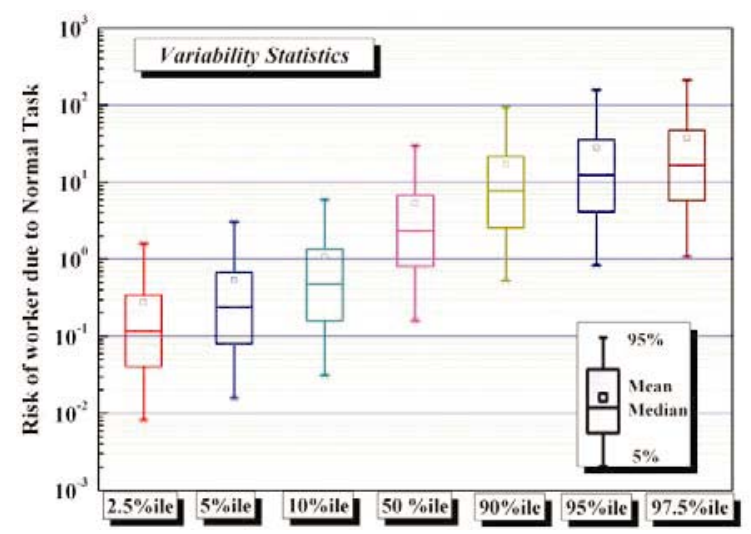

(b) worker

Fig. 7 The statistical summary for variability of radiological risk from 2D MCA due to normal task: (a) public, (b) worker The presented are $90 \%$ confidence intervals for each percentile.

public member and the worker due to normal task are given in Fig. 7.

\section{Conclusion}

The expert role provides valuable information through his or her decision within the framework of the availability of the data which is uncertain and restrictive, but certainly needed for risk analysis. In this study, the Delphi survey was tried out for obtaining the expert decisions, and the risks of the public and the workers were evaluated by the input characteristic. The approach characterizing input parameters using the Bayesian inference provided more improved risk estimates without intentional rejection of part of the data. The Bayesian updating of distributions of uncertain input parameters is then recommended as a useful tool in probabilistic risk analysis to provide more informative output. Also, it is expected that the results of this study can be used as a reference in developing the strategy for risk informed regulations over the field radiography practices.

\section{Acknowledgments}

This work was supported by Korean Ministry of Knowledge Economy (2008-P-EP-HM-E-06-0000), Ministry of Education, Science and Technology of Korea, and Sunkwang Atomic Energy Safety Co., Ltd..

\section{References}

1) R.T. Clemen and R.L. Winkler, Combining probability Distribution from Expert in Risk Analysis, risk Analysis, Vol. 19, No. 2, (1999).

2) Okoli C. Pawlowski SD. The delphi method as a Research Tool: an Example, Design Considerations and Applications, Information \&Management, Vol 42, (2004) 15-29.

3) J.S. Cullen and H.C. Frey, Probabilistic Techniques in Exposure Assessment; A Handbook for Dealing with Variability and Uncertainty in Models and Inputs. New York:Plenumpress, (1999).

4) Morgan M.G. and Herion M., Uncertainty; A Guide to Dealing with Uncertainty in Quantitative Risk and Policy Analyiss, Cambridge, UK: Cambridge University Press, (1990).

5) R. R. Lester, L.C. Green and Igor Linkov, Site-Specific Application of Probabilistic Health Risk Assessment: Review of the Literature Since 2000, Risk Analysis, Vol27(3) (2007) 635-654.

6) David L. Macintosh, Glenn W. Suter II, and F. Owen Hoffman, Uses of Probabilistic Exposure Models in Ecological Risk Assessments of Contaminated Sites, Risk Analysis 14(4) (1994) 405-419.

7) F. Owen Hoffman and Jana S. Hammonds, Propagation of Uncertainty in Risk Assessments: The Need to Distinguish Between Uncertainty Due to Lack of Knowledge and Uncertainty Due to Variability, Risk Analysis 24(5) (1994) 707-712.

8) Kimberly M. Thompson, Variability and Uncertainty Meet Risk Management and Risk Communication, Risk Analysis 22(3) (2002) 647-654.

9) Decisioneering Inc., CrystalBall@2000: User Manual, (2005).

10) Bilal M. Ayyub, Elicitation of Expert Opinions for Uncertainty and Risks, New York:CRC Press, (2001).

11) M.M. Meyer, J.M. Booker, Eliciting and Anlayzing Expert Judgment: A practical Guide, ASA SIAM Press, (2001).

12) Okoli C. Pawlowski SD., The Delphi method as a Research Tool: An Example, Design Considerations and Applications, Information \&Management, Vol 42 (2004) 15-29.

13) Spiegelhalter D, Thomas A, Best N, Lunn D, WinBUGS User Manual, Ver 1.4, (2003).

$<$ http://www.mrc-bsu.cam.ac.uk/bugs/>.

14) David L. Macintosh, Glenn W. Suter II, and F. Owen Hoffman, Uses of Probabilistic Exposure Models in Ecological Risk Assessments of Contaminated Sites, Risk Analysis 14(4) (1994) 405-419.

15) F. Owen Hoffman and Jana S. Hammonds, Propagation of Uncertainty in Risk Assessments: The Need to Distinguish Between Uncertainty Due to Lack of Knowledge and Uncertainty Due to Variability, Risk Analysis 24(5) (1994) 707-712.

16) Kimberly M. Thompson, Variability and Uncertainty Meet Risk Management and Risk Communication, Risk Analysis 22(3) (2002) 647-654. 